\title{
Randomized Trial of a Home-Based Family Intervention for Children Who Have Deliberately Poisoned Themselves
}

HARRINGTON, RICHARD FRCPsych; KERFOOT, MICHAEL PhD; DYER, ELIZABETH MRCPsych; McNIVEN, FAYE MSc; GILL, JULIA MSc; HARRINGTON, VALERIE BSc; WOODHAM, ADRINE MA; BYFORD, SARAH MSc

Accepted December 5, 1997.

From the Department of Child and Adolescent Psychiatry, Royal Manchester Children's Hospital, Pendlebury, Manchester, England (Professor Harrington); Department of Psychiatric Social Work, University of Manchester (Professor Kerfoot, Ms. McNiven, Ms. Gill, Ms. Harrington, Ms. Woodham); Department of Child Psychiatry, St. Mary's Hospital, Manchester (Dr. Dyer); and Centre for Health Economics, University of York, England (Ms. Byford).

This research was supported by the Department of Health, London. The authors thank the mental health teams who helped with this study and the children and families who gave so freely of their time.

Reprint requests to Professor Richard Harrington, Department of Child and Adolescent Psychiatry, Royal Manchester Children's Hospital, Pendlebury, Manchester M27 4HA, England; e-mail: r.c.harrington@man.ac.uk.

\section{ABSTRACT}

Objective: To establish whether an intervention given by child psychiatric social workers to the families of children and adolescents who had attempted suicide by taking an overdose reduced the patients' suicidal feelings and improved family functioning.

Method: One hundred sixty-two patients, aged 16 or younger, who had deliberately poisoned themselves were randomly allocated to routine care $(n=77)$ or routine care plus the intervention $(n=85)$. The intervention consisted of an assessment session and four home visits by the social workers to conduct family problem-solving sessions. The control group received no visits. Both groups were assessed at the time of recruitment and 2 and 6 months later. The primary outcome measures were the Suicidal Ideation Questionnaire, the Hopelessness Scale, and the Family Assessment Device.

Results: There were no significant differences in the primary outcomes between the intervention and control groups at either of the outcome assessments. Parents in the intervention group were more satisfied with treatment (mean difference 1.4 [95\% confidence interval 0.6 to 2.1]). A subgroup without major depression had much less suicidal ideation at both outcome assessments (analysis of covariance $\mathrm{p}<.01$ ) compared with controls.

Conclusions: The home-based family intervention resulted in reduced suicidal ideation only for patients without major depression. J. Am. Acad. Child Adolesc. Psychiatry, 1998, 37(5):512-518. 
Key Words: attempted suicide, self-poisoning, adolescent, child, randomized treatment trial.

Deliberate self-poisoning among children and adolescents is an important problem. In the United Kingdom, referrals to hospitals because of deliberate self-poisoning may be increasing and account for approximately $90 \%$ of all cases of deliberate self-harm (Hawton et al., 1996) [9]. In some health districts in the United Kingdom, adolescents who have taken a deliberate overdose account for one fifth of referrals to child psychiatrists (Kerfoot and McHugh, 1992) [17]. The risk of repetition of deliberate selfharm is approximately 10\% within 1 year (Spirito et al., 1989) [25]. One of the strongest correlates of completed suicide among the young is a previous attempt (Marttunen et al., 1993) [19].

There has been much research on the aftercare of young adults who have harmed themselves (Hawton, 1992) [7], but little is known about how this problem is best managed in children and adolescents. We know of only one published randomized trial in this age group, a study of the value of readmission to hospital on demand, which failed to find a significant effect of the intervention (Cotgrove et al., 1995) [5]. As far as we know there have been no published randomized trials of the psychosocial interventions that are commonly used by child mental health teams, such as family interventions and individual therapies.

We report a randomized trial of a brief home-based family intervention conducted by child psychiatric social workers with adolescents who have taken overdoses and their families. We chose a brief home-based program because previous work has shown that this group comply poorly with traditional hospital out-patient appointments and frequently fail to complete lengthy courses of treatment (Trautman et al., 1993) [26]. A family intervention was used because our previous research has shown a strong and specific association between deliberate self-poisoning in adolescence and family dysfunction (Kerfoot, 1988; Kerfoot et al., 1996) [14,15]. The two main hypotheses were (1) that the intervention would lead to lower levels of adolescent suicidal ideation and (2) that the families would function better.

\section{METHOD}

\section{Inclusion and Exclusion Criteria}

The study was based on a consecutive series of children and adolescents referred to child mental health teams working in four hospitals in Manchester, England. Patients were eligible if they were aged 16 years or younger with a diagnosis of deliberate selfpoisoning according to a standard definition (Hawton and Catalan, 1982) [8] and if they and their parents or legal guardian gave consent. Referrals with other forms of self-harm 
such as cutting or attempted hanging were excluded because it was felt they would require other interventions.

Young people deliberately poison themselves for a variety of reasons, and we wanted to target the group who were most likely to benefit from the intervention. Patients were therefore excluded if their social situation precluded a family intervention (e.g., if the child was not living in a family, if the child was in the care of social services, or if physical or sexual abuse was being investigated) or if a child psychiatrist decided there was a clinical or psychiatric contra-indication (e.g., if the child had severe mental illness such as psychosis, if the child was currently a psychiatric patient, or if the parent or child had significant learning difficulties or was severely suicidal). Cases in which it was unclear whether the overdose was deliberate or not (e.g., overdose of a drug usually used for kicks, or the adolescent denied that the overdose was deliberate) were also excluded because the intervention program assumes that the overdose is deliberate. Young people with major depression were not excluded because in a previous study in the same hospitals we had shown that major depression after a deliberate overdose resolved rapidly in most cases (Kerfoot et al., 1996) [15].

\section{Recruitment}

All patients referred to the child mental health teams were entered on a register and then assessed by a child psychiatrist and a child psychiatric social worker on the pediatric ward once they had recovered from the physical effects of the overdose. This brief initial assessment was used to determine eligibility for the study and was separate from the first family session. All the hospitals had a policy of admitting child overdose cases for an overnight stay. A series of opaque and sealed envelopes containing either a blank or the letter F were prepared and randomly assorted by an assistant. These were opened by the social worker at the time of the assessment, who then assigned the case to the family intervention plus routine care or routine care alone. Treatment assignment was entered on a register and concealed from the outcome assessors (V.H. and A.W.). The project was approved by the ethical committees of all the health districts taking part.

\section{Child Psychiatric Social Workers and Interventions}

Two master's-level child psychiatric social workers with previous child mental health experience (F.M. and J.G.) were employed specifically to conduct the intervention. They were trained by M.K., who met with them weekly for therapy supervision. The social workers also received supervision from a consultant child psychiatrist (E.D.).

The intervention program (Kerfoot, 1986; Kerfoot et al., 1995) [13,16] was devised by M.K. and was specified in a training manual and videotape. The essential features of the program were that it was short-term, intensive, focused, action-orientated, and homebased. The rationale behind it was that because self-poisoning in adolescents is strongly associated with family dysfunction (Hawton et al., 1996; Kerfoot, 1988; Kerfoot et al., 
1996) $[9,14,15]$, improvements in family functioning should lead to reduced suicidality in the child and to reduced stress within the family.

The program consisted of five sessions, the first occurring either in hospital or at home soon after the child had recovered from the overdose, and four at home. The first session consisted of an assessment and a conjoint interview to explain the program and negotiate goals. The second was concerned with discussing the episode itself. The third covered communication and the fourth, problem-solving. The final session was concerned with some of the developmental issues of adolescence and their impact on the family. All members of the family were encouraged to attend, but to count as a session the adolescent and at least one parent had to be present.

As part of a health economic evaluation conducted by S.B., routine psychiatric aftercare was carefully audited using standardized forms. It comprised visits to the clinic by the adolescent and family for an average of 3.2 sessions ( $\mathrm{SD}=4.4$ sessions) in the intervention group and 3.6 sessions in the control group ( $\mathrm{SD}=4.0$ sessions). A diverse range of interventions, including sessions with psychiatrists and with psychiatric nurses, was administered across the four hospitals, but none used a home-based family intervention.

\section{Assessments}

The primary outcome measures were validated in a pilot study conducted by the two outcomes assessors (Kerfoot et al., 1996) [15] and comprised the Suicidal Ideation Questionnaire (Reynolds, 1988) [22] and the Hopelessness Questionnaire (Kazdin et al., 1986) [12] completed by the patients, and a questionnaire measure of family functioning completed independently by both the patient and the parent(s) (Miller et al., 1985) [20].

Secondary outcome measures were the Generation of Alternative Solutions subscale of the Social Problem Solving Inventory (Sadowski and Kelley, 1991) [24], which was completed by the child; the 28-item version of the General Health Questionnaire (Goldberg, 1978) [6], a measure of stress completed by the parent(s); and 8-point Likert scales of satisfaction with treatment completed by the child and parent(s) (Wood et al., 1996) [27]. The children's and parents' expectancies of treatment were also assessed on a 0 to 8 Likert scale.

The outcome assessors interviewed the child with portions of the Schedule for Affective Disorders and Schizophrenia for School-Age Children (Ambrosini, 1988) [1] to diagnose major depression, conduct disorder, and substance abuse according to DSM-IV criteria (American Psychiatric Association, 1994) [2]. Parents were interviewed with the Deliberate Self-Harm Interview Schedule (DSHIS) to establish current family circumstances and details of school performance (Kerfoot, 1988) [14]. Our pilot study showed that the DSHIS had good discriminant validity. Efforts were made to keep the assessors blind to case-control status, but this was difficult because the home-based family intervention was so different in character from routine care, which usually 
involved individual sessions given at the hospital. When asked to guess which treatment had been given, the assessors guessed correctly $(\mathrm{n}=92)$ more often than incorrectly $(\mathrm{n}=$ 70 ), though this did not reach statistical significance (chi squared $=3.0, \mathrm{p}=.08$ ).

\section{Target Sample Size}

This study was not designed to examine the impact of the intervention on rates of repetition but rather on suicidal thinking. A pilot study in the same hospitals (Kerfoot et al., 1996) [15] showed that after routine aftercare, the mean Suicidal Ideation Questionnaire score (mean $=36.2, \mathrm{SD}=26.3$ ) was still much higher than in matched community controls (mean $=8.8, \mathrm{SD}=16.9$ ). In line with other research (Jacobson and Truax, 1991) [11], an empirical decision was made that clinically significant change would be defined as a mean level of symptomatology after active treatment that was more than halfway between the mean score after the control treatment and the mean score for the general population. We therefore decided that we needed to detect a mean Suicidal Ideation Questionnaire score of 24.0 after active treatment, giving an effect size of 0.46 (36.2 minus 24.0, divided by the SD, 26.3). This is considered a medium effect in psychotherapy research (Cohen, 1992) [4], and it meant that the target sample size was 79 in each group for $80 \%$ power and .05 significance (Machin and Campbell, 1987) [18].

\section{Analysis}

Analyses were conducted with the statistical package SPSS. No stoppage rules were applied, and therefore just one analysis was conducted at the end of the study. All the analyses were conducted "intent to treat." Initially, all continuous variables were subjected to distributional analysis and appropriate normalizing transformations were sought for measures found not to follow a normal distribution. Then, equality of the groups on pretreatment data was determined. Differences between groups on continuous measures were tested by analysis of covariance with the pretreatment score as a covariate. Separate analyses were conducted according to whether or not the assessors had guessed the treatment group correctly, but this made no material difference to the results.

\section{RESULTS}

\section{Progress of the Trial}

During the 27-month recruitment phase, a total of 435 young people aged 16 or younger (range 10 to 16 years) who had taken an overdose were referred to the child psychiatry teams. Thirty-eight cases were excluded because they had not taken a deliberate overdose, 48 because they had a psychiatric contraindication, and 61 cases because their social situation precluded a family intervention. One hundred nine either refused further treatment or refused to enter the trial, or both. Contact was lost with 17 (e.g., absconded from the ward, ran away from home). One hundred sixty-two cases were recruited to the 
trial and then randomly allocated to either routine care and the home-based family intervention $(n=85)$ or routine care alone $(n=77)$.

There was good compliance with the family intervention, with only 22 (26\%) of 85 cases missing one or more of the five sessions. Compliance with routine care was less good, with at least one missed hospital appointment occurring in 45 (54\%) of 84 cases in the intervention group and 49 (64\%) of 77 cases in the control group. Outcome assessments were conducted with 154 (96\%) of 162 cases at 2 months and 149 (92\%) of 162 cases at 6 months.

\section{Patients}

The groups were well matched on clinical characteristics (Table 1). In line with our previous studies (Kerfoot, 1988; Kerfoot et al., 1996) [14,15], the children tended to come from deprived backgrounds and were often in conflict with their families. They had a high rate of major depression.

[Help with image viewing] [Email Jumpstart To Image]
Table 1. Clinical Features of Patients in the Intervention Group and Control Group Before Treatment

\section{Primary Outcomes}

The groups were well matched on the primary outcomes at the pretreatment assessment. There were no significant differences between the groups at either of the follow-up assessments (Table 2).

[Help with image viewing] [Email Jumpstart To Image]
Table 2. Primary Outcome Measures for Patients in the Intervention Group and Control Group

\section{Secondary Outcomes}


The only significant difference between the groups with respect to the secondary outcomes was that parents in the family intervention group were significantly more satisfied with treatment at the 2-month assessment (Table 3). This difference remained significant even when expectancy about treatment was entered as a covariate in an analysis of covariance.

[Help with image viewing] [Email Jumpstart To Image]
Table 3. Secondary Outcome Measures for Patients in the Intervention Group and Control Group

Subgroup Analysis

We have previously found in a randomized trial (Wood et al., 1996) [27] that children and adolescents with major depression improve with individual cognitive-behavioral therapy. We were therefore particularly interested in whether the family intervention would help overdose cases who were not depressed, because no intervention has yet been proven to help this group. There was a significant interaction between treatment group, major depression, and suicidal ideation. Thus, for example, in a simple factorial analysis of variance with suicidal ideation at 2 months as the dependent variable and treatment group and major depression at baseline as factors, there was no significant main effect of treatment group, a significant effect of major depression $(\mathrm{F}=15.9, \mathrm{p}<.001)$, and a significant two-way interaction between treatment group and major depression $(\mathrm{F}=4.2, \mathrm{p}$ $=.04)$.

Separate analyses were therefore performed within the depressed and nondepressed subgroups. There were no significant effects of treatment within the depressed subgroup, but within the nondepressed sample the intervention group had much less suicidal ideation than the control group at both the 2-month and the 6-month assessments (Table 4). With suicidal ideation before treatment as a covariate, the effect of treatment was significant at both 2 months $(\mathrm{df}=2,49, \mathrm{~F}=8.7, \mathrm{p}<.01)$ and 6 months $(\mathrm{df}=2,48, \mathrm{~F}=8.6$, $\mathrm{p}<.01$ ). There were no treatment effects on the other primary outcome variables within this subgroup.

[Help with image viewing] [Email Jumpstart
Table 4. Suicidal Ideation in the Intervention Group and Control Group for Patients Without Major Depression Before Treatment 
To Image]

\section{DISCUSSION}

The aftercare of young people who have deliberately poisoned themselves is an important clinical issue for child mental health teams, yet little is known about the best ways of helping these children and their families. This study examined the efficacy of a brief home-based family intervention delivered by child psychiatric social workers.

The main finding was that social worker visits did not result in a better outcome for children who had taken an overdose or for their families. A moderate treatment effect on suicidal ideation was observed in the subgroup who did not have major depression. The clinical significance of this result is limited, however, by the finding that young people who harm themselves but who are not depressed are at relatively low risk of subsequent problems (Pfeffer et al., 1993) [21]. Indeed, in our study this subgroup had less suicidal ideation than depressed overdose cases.

\section{Family Intervention}

The family intervention targeted difficulties such as poor communication and impaired problem-solving, which we have previously shown in the same population using the same measures are particularly prevalent in overdose cases compared both with normal controls and with other nonsuicidal child psychiatric patients (Kerfoot et al., 1996) [15]. The intervention has been used for many years (Kerfoot, 1986) [13], and in the present study it was delivered by child psychiatric social workers who were employed specifically for the study. It was popular with the parents, and there was better compliance with it than with routine care. Compliance was also better than has been reported in other studies of children who have harmed themselves (Trautman et al., 1993) [26].

It may be, however, that some family problems cannot be resolved by just five sessions and require more therapy. Indeed, the finding that our main measure of family functioning, the Family Assessment Device, did not change during the study suggests that our treatment may not have done what it was supposed to do. Also, a specialized family intervention may not address the diversity of problems that a child's overdose can represent. Although efforts were made to include only those patients who were most likely to be helped by the intervention, this was a pragmatic trial with a heterogeneous population including patients with major depression and conduct disorder. The failure to find an effect of a family intervention within the depressed subgroup is consistent with the results of Brent and colleagues (1997) [3], who reported that family therapy was not significantly better than a control intervention in adolescents with major depression. It is also consistent with the findings from another study of adolescents who have harmed themselves that the initial level of symptoms is significantly associated with the impact of 
a treatment program (M.J. Rotheram-Borus, J. Piacentini, C. Cantwell, T.R. Belin, J. Song, unpublished, 1997).

\section{Trial Design}

Several other factors might have contributed to the failure to find a significant difference between the groups. We followed up slightly fewer than the 79 cases in each group that we had targeted on the basis of our power calculations. Moreover, because it was necessary that there be professional contact between the research social workers and the mental health workers who were giving routine care, it is possible that some parts of the intervention became incorporated into routine care (however, we have no evidence from our audit that this had occurred). Also, there might have been a better response to the intervention in those who refused further treatment, although this seems improbable.

\section{Clinical Implications}

A conjoint family intervention aimed at reducing suicidal thinking in children and adolescents who had taken a deliberate overdose was no more effective than routine care. There seemed to be an effect of treatment within the subgroup who did not have a depressive disorder. However, this finding needs confirmation in further studies in which there is a priori stratification by depression. In the meantime, other interventions such as cognitive-behavioral family approaches (Rotheram-Borus et al., 1994) [23] or individual therapies may be indicated in children who have taken an overdose.

In both the United Kingdom and the United States, there has been a growing demand for psychiatric treatments to be made more accessible to children and their families. For instance, in a report that has been very influential in the United Kingdom, the National Health Service Health Advisory Service put accessibility at the top of the list of principles of providing child and adolescent mental health services (Health Advisory Service, 1995) [10]. Our findings show, however, that easy access to a treatment is no guarantee that it works.

\section{REFERENCES}

1. Ambrosini PJ (1988), Schedule for Affective Disorders and Schizophrenia for SchoolAge Children (6-18 Years). New York: New York State Psychiatric Institute. [Context Link]

2. American Psychiatric Association (1994), Diagnostic and Statistical Manual of Mental Disorders, 4th edition (DSM-IV). Washington, DC: American Psychiatric Association. [Context Link] 
3. Brent D, Holder D, Kolko D et al. (1997), A clinical psychotherapy trial for adolescent depression comparing cognitive, family, and supportive treatments. Arch Gen Psychiatry 54:877-885. ExternalResolverBasic Bibliographic Links [Context Link]

4. Cohen J (1992), A power primer. Psychol Bull 112:155-159. [Context Link]

5. Cotgrove A, Zirinsky L, Black D, Weston D (1995), Secondary prevention of attempted suicide in adolescence. J Adolesc 18:569-577. ExternalResolverBasic Bibliographic Links [Context Link]

6. Goldberg D (1978), General Health Questionnaire. Windsor, England: NFER-Nelson. [Context Link]

7. Hawton K (1992), Suicide and attempted suicide. In: Handbook of Affective Disorders, 2nd ed, Paykel ES, ed. Edinburgh: Churchill Livingstone, pp 635-650. [Context Link]

8. Hawton K, Catalan J (1982), Attempted Suicide. Oxford, England: Oxford Medical Publications. [Context Link]

9. Hawton K, Fagg J, Simkin S (1996), Deliberate self-poisoning and self-injury in children and adolescents under 16 years of age in Oxford, 1976-1993. Br J Psychiatry 169:202-208. ExternalResolverBasic Bibliographic Links [Context Link]

10. Health Advisory Service (1995), Child and Adolescent Mental Health Services. London: HMSO. [Context Link]

11. Jacobson NS, Truax P (1991), Clinical significance: a statistical approach to defining meaningful change in psychotherapy research. J Consult Clin Psychol 59:12-19. [Context Link]

12. Kazdin AE, Rodgers A, Colbus D (1986), The Hopelessness Scale for Children: psychometric characteristics and concurrent validity. J Consult Clin Psychol 54:241-245. Ovid Full Text ExternalResolverBasic Bibliographic Links [Context Link]

13. Kerfoot M (1986), Family therapy and psychotherapy following suicidal behaviour by young adolescents. In: Suicide in Adolescence, Diekstra R, Hawton K, eds. Dordrecht, Netherlands: Martinus Nijhoff, pp 95-111. [Context Link]

14. Kerfoot M (1988), Deliberate self-poisoning in childhood and early adolescence. J Child Psychol Psychiatry 29:335-345. ExternalResolverBasic Bibliographic Links [Context Link]

15. Kerfoot M, Dyer E, Harrington V, Woodham A, Harrington RC (1996), Correlates and short-term course of self-poisoning in adolescents. Br J Psychiatry 168:38-42. ExternalResolverBasic Bibliographic Links [Context Link] 
16. Kerfoot M, Harrington RC, Dyer E (1995), Brief home-based intervention with young suicide attempters and their families. J Adolesc 18:557-568. ExternalResolverBasic Bibliographic Links [Context Link]

17. Kerfoot M, McHugh B (1992), The outcome of childhood suicidal behaviour. Acta Paedopsychiatr 55:141-145. ExternalResolverBasic Bibliographic Links [Context Link]

18. Machin D, Campbell MJ (1987), Statistical Tables for the Design of Clinical Trials. Oxford, England: Blackwell. [Context Link]

19. Marttunen MJ, Aro HM, Lonnqvist JK (1993), Adolescence and suicide: a review of psychological autopsy studies. Eur Child Adolesc Psychiatry 2:10-18.

ExternalResolverBasic Bibliographic Links [Context Link]

20. Miller IV, Epstein NB, Bishop DS, Keitner GB (1985), The McMaster Family Assessment Device: reliability and validity. J Marital Fam Ther 11:345-356.

ExternalResolverBasic [Context Link]

21. Pfeffer CR, Klerman GL, Hurt SW, Kakuma T, Peskin JR, Siefker CA (1993), Suicidal children grow up: rates and psychosocial risk factors for suicide attempts during follow-up. J Am Acad Child Adolesc Psychiatry 32:106-113. ExternalResolverBasic Bibliographic Links [Context Link]

22. Reynolds WM (1988), Suicidal Ideation Questionnaire: Professional Manual. Odessa, FL: Psychological Assessment Resources. [Context Link]

23. Rotheram-Borus MJ, Piacentini J, Miller S, Graae F, Castro-Blanco D (1994), Brief cognitive-behavioral treatment for adolescent suicide attempters and their families. J Am Acad Child Adolesc Psychiatry 33: 508-517. ExternalResolverBasic Bibliographic Links [Context Link]

24. Sadowski C, Kelley ML (1991), Social problem solving in suicidal adolescents. J Consult Clin Psychol 61:121-127. [Context Link]

25. Spirito A, Brown L, Overholser J, Fritz G (1989), Attempted suicide in adolescence: a review and critique of the literature. Clin Psychol Rev 9:335-363.

ExternalResolverBasic Bibliographic Links [Context Link]

26. Trautman PD, Stewart N, Morishima A (1993), Are adolescent suicide attempters noncompliant with outpatient care? J Am Acad Child Adolesc Psychiatry 32:89-94. ExternalResolverBasic Bibliographic Links [Context Link]

27. Wood AJ, Harrington RC, Moore A (1996), Controlled trial of a brief cognitivebehavioral intervention in adolescent patients with depressive disorders. J Child Psychol Psychiatry 37:737-746. ExternalResolverBasic Bibliographic Links [Context Link] 
Accession Number: 00004583-199805000-00014

Copyright (c) 2000-2006 Ovid Technologies, Inc.

Version: rel10.2.2, SourceID 1.11354.1.251 